\title{
包围型催化剂的逆向设计合成及催化性能
}

侯文华

南京大学化学化工学院, 介观化学教育部重点实验室, 江苏南京 210023

\section{Surrounded Catalysts: The Inverse Design, Preparation and Catalytic Performance}

\section{Wenhua Hou}

State Key Laboratory of Mesoscopic Chemistry of MOE, School of Chemistry and Chemical Engineering, Nanjing University, Nanjing 210023, China.

Email: whou@nju.edu.cn

Published online: August 24, 2020.
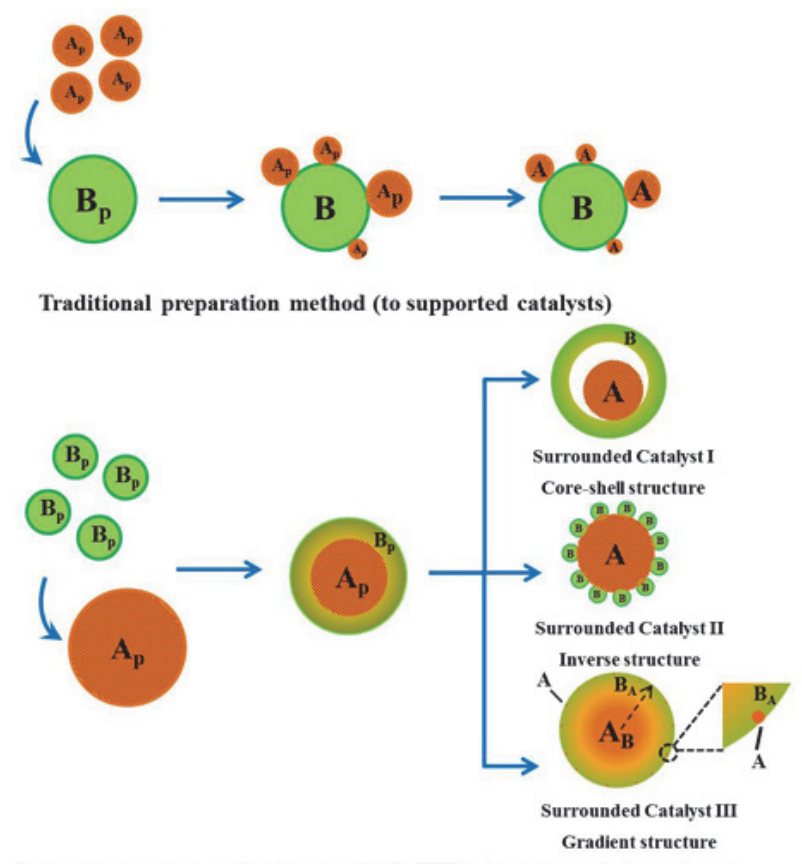

Ion-exchange inverse loading method (IEIL) (to surrounded catalysts)
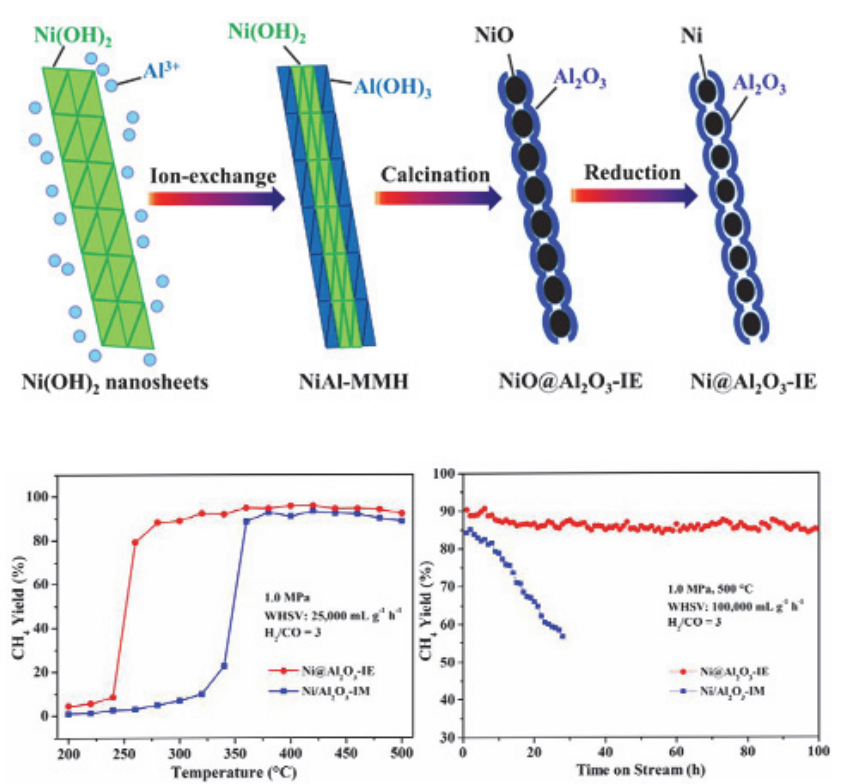

传统负载型催化剂及包围型催化剂的制备示意图和 $\mathrm{Ni} @ \mathrm{Al}_{2} \mathrm{O}_{3}$-IE 包围型催化剂合成过程示意图及催化性能。

负载型催化剂, 通常是催化活性相负载在比 表面积较大的载体上, 在多相催化过程中被广泛 使用 ${ }^{1}$ 。很多情况下, 载体不仅是用来分散催化活 性相/颗粒, 它也可以通过与活性相之间的作用来 调控活性相的电子及几何结构, 参与并影响催化 反应过程 2 。自 20 世纪 70 年代, Tauster等人 3 提出金 属-载体强相互作用(SMSI) 以来, 越来越多的研究 关注到活性相和载体之间的界面相互作用及界面
密度对催化反应机制及催化性能的影响。例如, 包 信和团队4通过巧妙设计, 在贵金属表面构筑高分 散的过渡金属氧化物形成反相结构, 使金属-氧化 物界面密度最大化, 而界面提供的独特化学环境 对活性中心产生限域效应, 维持其配位不饱和性, 并在低温催化氧化反应中表现出优越的催化性 能。郑南峰团队 5 采用介孔 $\mathrm{SiO}_{2}$ 包覆层状硅酸铜纳 米管, 形成富含 $\mathrm{Cu}-\mathrm{O}-\mathrm{SiO}_{x}$ 界面活性位点的催化 
剂, 促成 $\mathrm{H}_{2}$ 异裂, 并通过稳定过渡态促进酯加氢, 使其在草酸二甲酯加氢制乙二醇中表现出优异性 能。路军岭团队6通过采用原子气相沉积法原子级 精准地构筑出单位点 $\mathrm{Fe}_{1}(\mathrm{OH})_{x}$ 物种, 并针定在 $\mathrm{Pt}$ 纳 米颗粒上, 获得了丰富 $\mathrm{Fe}_{1}(\mathrm{OH})_{x}$ - $\mathrm{Pt}$ 单位点界面催 化活性中心, 并在CO优先氧化(PROX)反应中, 超 宽温度区间内, 成功实现了 $\mathrm{CO}$ 的 $100 \%$ 选择性完全 去除。从上述研究报道可以看出, 界面位点在催化 反应中起着越来越重要甚至是主导作用, 而提高 界面相互作用及界面密度/面积成为提升催化性能 的有效途径。

传统负载型催化剂主要通过浸渍法或沉淀法 制备 ${ }^{7}$ 。在这些方法中, 活性金属物种的前驱体通 常沉积在载体的外表面, 使得活性物种与载体之 间的界面相互作用较弱且界面密度有限, 在催化 剂后处理或反应过程中, 导致活性物种团聚, 进一 步减小界面密度, 影响催化性能。因此, 发展新的 催化剂制备方法, 设计制备具有丰富界面位点的 催化剂结构对于提升负载型催化剂性能具有重要 意义。

近期, 南京大学郭学锋教授和丁维平教授研 究团队提出反向设计构造包围型催化剂的设想, 并发展了一种简易且普适的包围型催化剂制备方 法, 即离子交换逆负载(IEIL)法制备包围型催化 剂。相关研究成果已于近期发表在 Science Advances 上。 $^{8}$.

在IEIL法(如图所示)中, 活性金属氢氧化物前 体 $\left(\mathrm{A}_{\mathrm{p}}\right)$ 投入到载体前体 $\left(\mathrm{B}_{\mathrm{p}}\right)$ 的溶液中, 在氢氧化物 溶度积的差异下发生离子交换反应, 载体前体的 金属离子取代 $\mathrm{A}_{\mathrm{p}}$ 表面的金属离子形成混合金属氢 氧化物, 随后经焙烧、还原形成具有核壳结构的 $\mathrm{A} @ \mathrm{~B}$ 包围型催化剂。例如 $\mathrm{A}_{\mathrm{p}}$ 代表 $\mathrm{Ni}(\mathrm{OH})_{2}$ 纳米片, 用 $\mathrm{Al}^{3+}$ 大量交换 $\mathrm{Ni}^{2+}$, 最终得到具有核壳结构的 $\mathrm{Ni} @ \mathrm{Al}_{2} \mathrm{O}_{3}$-IE包围型催化剂。调控不同的离子交换 程度及金属、载体前驱体的种类, 还可得到具有反
相结构、梯度结构等多种包围型催化剂。相比于传 统负载型催化剂, 包围型结构具有更高的界面密 度和相互改变的界面性质, 并且因为活性相被载 体物理隔离具有高稳定性, 使其在催化反应中表 现出更优越的催化性能。例如, $\mathrm{Ni} @ \mathrm{Al}_{2} \mathrm{O}_{3}$-IE包围 型催化剂在 $\mathrm{CO}$ 甲烷化反应中表现出优异的低温 活性及高温稳定性。

这项工作提出了反向设计合成活性核中心被 载体包围的高性能催化剂结构, 即 “包围型催化 剂”, 定义了一种活性核被载体包围同时具有相互 改变的界面的催化剂结构, 这种新的包围型催化 剂有望在多相催化领域成为传统负载型催化剂的 升级版, 进而推动工业催化技术进步。

\section{References}

(1) Mizuno, N.; Misono, M. Chem. Rev. 1998, 98, 199. doi: $10.1021 / \mathrm{cr} 960401 \mathrm{q}$

(2) Fu, Q.; Yang, F.; Bao, X. H. Acc. Chem. Res. 2013, 46, 1692. doi: $10.1021 / \operatorname{ar} 300249 \mathrm{~b}$

(3) Tauster, S. J.; Fung, S. C.; Garten, R. L. J. Am. Chem. Soc. 1978, 100, 170. doi: $10.1021 / \mathrm{ja} 00469 \mathrm{a} 029$

(4) Fu, Q.; Li, W. X.; Yao, Y. X.; Liu, H. Y.; Su, H. Y.; Ma, D.; Gu, X. K.; Chen, L. M.; Wang, Z.; Zhang, H.; et al. Science 2010, 328, 1141. doi: $10.1126 /$ science. 1188267

(5) Xu, C. F.; Chen, G. X.; Zhao, Y.; Liu, P. X; Duan, X. P.; Gu, L.; Fu, G.; Yuan, Y. Z.; Zheng, N. F. Nat. Commun. 2018, 9, 3367. doi: 10.1038/s41467-018-05757-6

(6) Cao, L. N.; Liu, W.; Luo, Q. Q.; Yin, R. T.; Wang, B.; Weissenrieder, J.; Soldemo, M.; Yan, H.; Lin, Y.; Sun, Z. H.; et al. Nature 2019, 565, 631. doi: 10.1038/s41586-018-0869-5

(7) Munnik, P.; Jongh, P. E.; Jong, K. P. Chem. Rev. 2015, 115, 6687. doi: $10.1021 / \mathrm{cr} 500486 \mathrm{u}$

(8) Hao, P. P.; Xie, M. J.; Chen, S. Y.; Li, M. H.; Bi, F. F.; Zhang, Y.; Lin, M.; Guo, X. K.; Ding, W. P.; Guo, X. F. Sci. Adv. 2020, 6, eaay7031. doi: $10.1126 /$ sciadv.aay 7031 